\title{
A Coronal Jet Ejection from a Sunspot Light Bridge
}

\author{
S. Liu \\ National Astronomical Observatory, Chinese Academy of Sciences, Beijing, China \\ Email: lius@nao.cas.cn
}

\begin{abstract}
Chromospheric brightening and $\mathrm{H} \alpha$ surges are evident and common phenomena along sunspot light bridges. In this paper, a coronal jet ejection from a sunspot light bridge is presented. Using data from the Solar Dynamics Observatory and Hinode satellites, it is confirmed that the jet has its root near the light bridge. This suggests that the jet may be a result of reconnection between the main sunspot and the light bridge. Due to the processing of jet ejecta, the intensity and width of the light bridge show changes to some extent. This also suggests that the jet is related to the interaction between light bridge and umbra, possibly owing to magnetic reconnection or heated plasma trapped in the light bridge escaping and moving along field lines.
\end{abstract}

Keywords: Sun: activity — Sun: corona — sunspots

Received 2011 December 29, accepted 2012 March 13, published online 2012 April 4

\section{Introduction}

Light bridges (LBs) are bright structures crossing the umbra during the evolution of sunspots. LBs are associated with the break-up of sunspots in decay or the assembly of sunspots in complex active regions (Bray \& Loughhead 1964; Vasquez 1973; Garcia de La Rosa 1987). Muller (1979) classified LBs as 'photospheric', 'penumbral' and 'umbral' according to their intensity and fine structure. Another classification is as follows (Sobotka, Bonet \& Vazquez 1993, 1994): strong LBs, which separate the umbral core and are further distinguished as photospheric or penumbral, and faint LBs, which are faint narrow lanes within the umbra and most likely consist of umbral dots.

At present, the formation and magnetic properties of LBs are not understood completely. A common mechanism to explain the formation of LBs is that field-free convection penetrates the umbra from the subphotosphere and forms a cusp-like magnetic field (Spruit \& Scharmer 2006). Katsukawa et al. (2007) revealed the formation of a LB due to the intrusion of umbral dots based on data obtained from the Hinode satellite. The magnetic field in a $\mathrm{LB}$ is revealed to be weaker and more inclined than that of the surrounding umbra (Ruedi, Solanki \& Livingston 1979; Leka 1997; Jurcak, Pillet \& Sobotka 2006). Based on Hinode observations of the magnetic field in a LB accompanied by long-lasting chromospheric plasma ejections, Shimizu et al. (2009) suggest that currentcarrying highly twisted magnetic flux tubes are trapped below a cusp-shaped magnetic structure along the LB. In addition, by a detailed analysis of the Stokes spectra (Jurcak et al. 2006) it is found that the field strengths and inclinations increase and decrease with height, which suggests a canopy-like structure above the LB.
It is indisputable that the plasma contained in a LB has a temperature higher than that in the surrounding umbra because of the brightness of the LB. Observations indicate that there are remarkable plasma ejections or $\mathrm{H} \alpha$ surge activity in the chromosphere along a LB (Roy 1973; Asai, Ishii \& Kurokawa 2001; Bharti et al. 2007; Shimizu et al. 2009). Additionally, over the site of a LB a bright enhancement in 1600- $\AA$ images and heating of coronal loops in 171- $\AA$ images from the Transition Region and Coronal Explorer (TRACE) was found recently (Berger \& Berdyugina 2003; Katsukawa 2007), which suggests that LBs are steady heat sources in the chromosphere. Louis, Bayanna \& Mathew (2008), using G-band and Ca II images obtained from Hinode, studied the dynamics and brightness enhancements of LBs and pointed to the possibility that LBs could be sites for heating the overlying chromosphere, but cannot rule out the likelihood of coronal phenomena. In this paper, a coronal jet originating from the site of a LB is presented, which means that the interaction between LB and umbra can also create coronal dynamic activity.

This paper is organized as follows: a description of observations and data used is introduced in Section 2, the results are shown in Section 3, and finally a short discussion and conclusions are given in Section 4.

\section{Observations and Data Reduction}

The jet studied here is near the disk center (heliographic coordinates S17W23) and occurred during about 20:00 21:00 UT on 2011 March 29. The observatory data used to study this jet were obtained by the Atmospheric Imaging Assembly (AIA: Title 2010; Boerner et al. 2010) and Helioseimic and Magnetic Imager (HMI: Schou et al. 2010) on board the Solar Dynamics Observatory (SDO) 

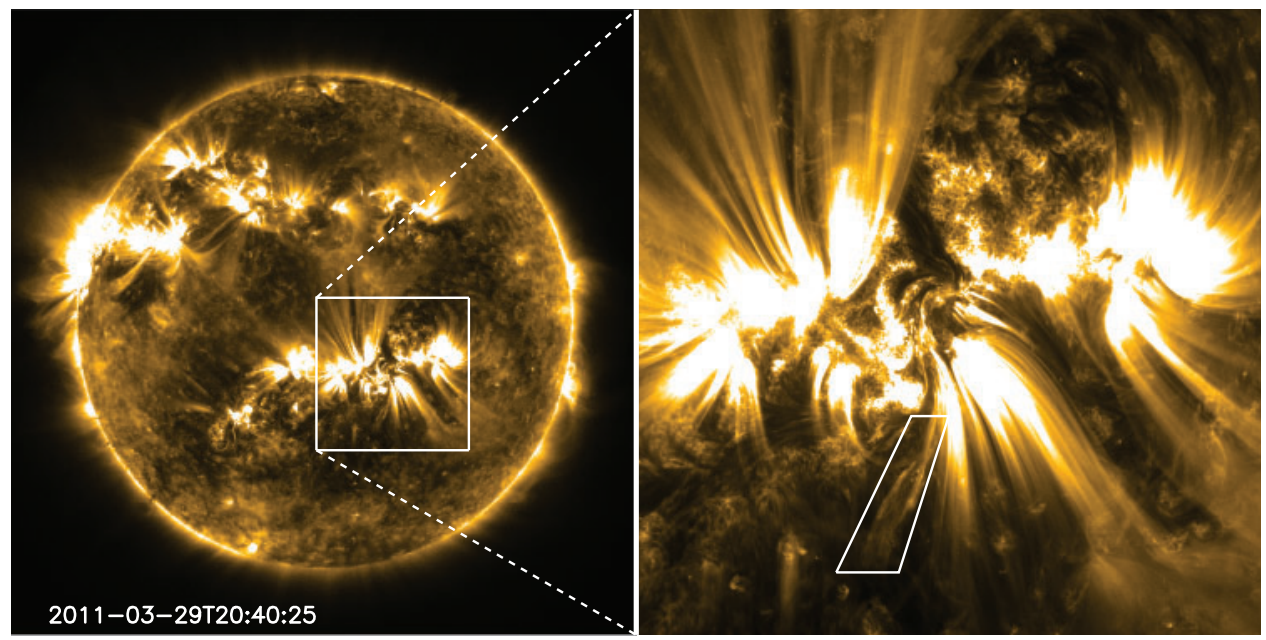

Figure 1 Left: the full-disk AIA 171 image at 20:40:25 UT on 2011 March 29. Right: a subregion drawn from the left image, where the coronal jet is highlighted by a rectangle.

and by the Solar Optical Telescope (SOT) on board Hinode (Kosugi et al. 2007; Tsuneta et al. 2008). The AIA takes full-disk images at 10 wavelengths with a pixel size of 0.59 arcsec. In this paper, AIA $171-\AA$ and $1700-\AA$ data at Level 1 with a 45 -s cadence are used. The HMI includes full-disk magnetograms, continuum intensities and dopplergrams with a spatial resolution of $0.5 \mathrm{arcsec}$. In this paper, line-of-sight (LOS) magnetograms and continuum intensity are used for the analysis. G-band and $\mathrm{Ca}$ II data (with a spatial resolution of 0.1 arcsec) and LOS magnetograms (with spatial and temporal resolution 0.16 arcsec and $15 \mathrm{~min}$, respectively) observed by SOT/Hinode are used in this work. The data processing in this work is all based on standard solar software (SSW, e.g. fg_prep.pro, aia_prep.pro). For example, dark-subtraction, flat-fielding, the correction of bad pixels, and cosmic-ray removal were performed for filtergram images obtained by SOT.

\section{Results}

Figure 1 shows the location of the jet, which occurs during about 20:00-21:00 UT on 2011 March 29. The left image is the full-disk AIA 171- $\AA$ image at 20:40 UT, and the region where the jet occurs is shown by a white rectangle, while the right image is an amplified subregion of the left image in which the jet (highlighted by a white region) can be seen clearly. It is found that the jet has already separated into two parts along its direction of propagation as time goes on, the former part ejected toward space and the latter one falling back along the magnetic field lines where it created.

Figure 2 shows the process of the coronal jet from 20:00-21:07 UT, with a peak at 20:45 UT using the AIA $171-\AA$ images. The field of view is 162 arcsec for all images. The blue arrow in each frame points to the evolution of this jet, including the start ((a) and (b)), maximum ((c) and (d)) and decay ((e) and (f)) phases. In Figure 2(d) the jet has already separated into two parts along its direction of propagation: the former part (yellow arrow) is ejected toward space away from the Sun, while the latter (blue arrow) becomes a back-flow along the magnetic field lines and gives rise to an intensity enhancement of the site where the jet originated, which can be seen in image (e) indicated by a red arrow. The velocity of back-flow is about $198 \mathrm{~km} \mathrm{~s}^{-1}$, which is calculated from the AIA 171- $\AA$ images; however the effect of projection is not considered when the velocity is calculated. To see the intensity enhancement resulting from the backflow, Figure 3 shows the evolution of 1700 - $\AA$ images from 20:53-20:59 UT. The intensity enhancement indicated by the blue arrow in panel (e) should be caused by the backflow corresponding to the latter part of jet.

In order to know the circumstances of the lower atmosphere corresponding to the site where the jet was created, Figure 4 gives the AIA (a) $171-\AA$ and (b) $1700-\AA$ images, (c) HMI LOS magnetogram and (d) continuum intensity images and (e) G-band and (f) Ca II SOT/Hinode images together. These images are aligned by heliospheric coordinates and combined by correlation of feature points (cross-correlation function IDL). The contour lines plotted on each image are LOS magnetic field, where the levels of magnetic field contours are -80 to -50 and 200-800 G, and red/green contours are positive/negative magnetic flux, while in Figure 4(f) the yellow contour is positive magnetic flux and the blue rectangle indicates the site where the jet originated. From the continuum intensity, G-band and 1700- $\AA$ images, it can be seen clearly that the jet has its root near the LB, which can be seen from the intensity enhancement of Figure 4(b) and (f) indicated by a white arrow. This intensity enhancement is due to mass falling back along magnetic field lines. Additionally, from Figure 4(c) it can be seen that there is no evident negative magnetic flux, which opposes the main positive magnetic flux near to the root of the jet. To see the fine structure of the magnetic field on the photosphere associated with jet eruption more clearly, the high spatial resolution ( 0.16 arcsec) LOS magnetic field observed by SOT/Hinode is shown in Figure 5; here the field of view is $40 \times 40 \operatorname{arcsec}^{2}$. The levels of magnetic field contours are 

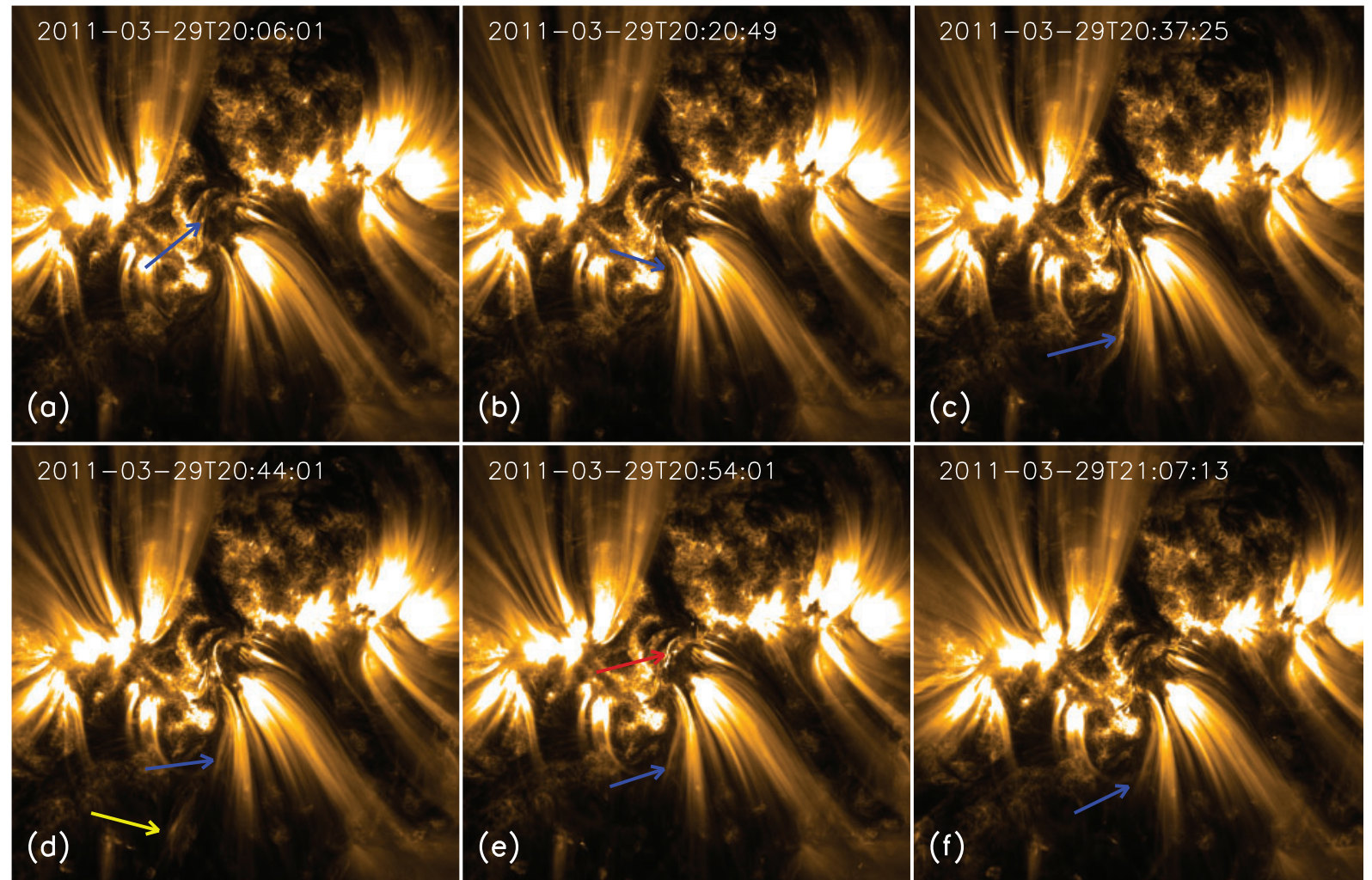

Figure 2 Time-series images of the AIA 171 channel showing the jet process. These includes the start ((a) and (b)), maximum ((c) and (d)) and decay ((e) and (f)) phases. It can be seen in (d) that the jet has already separated into two parts along its direction of propagation.

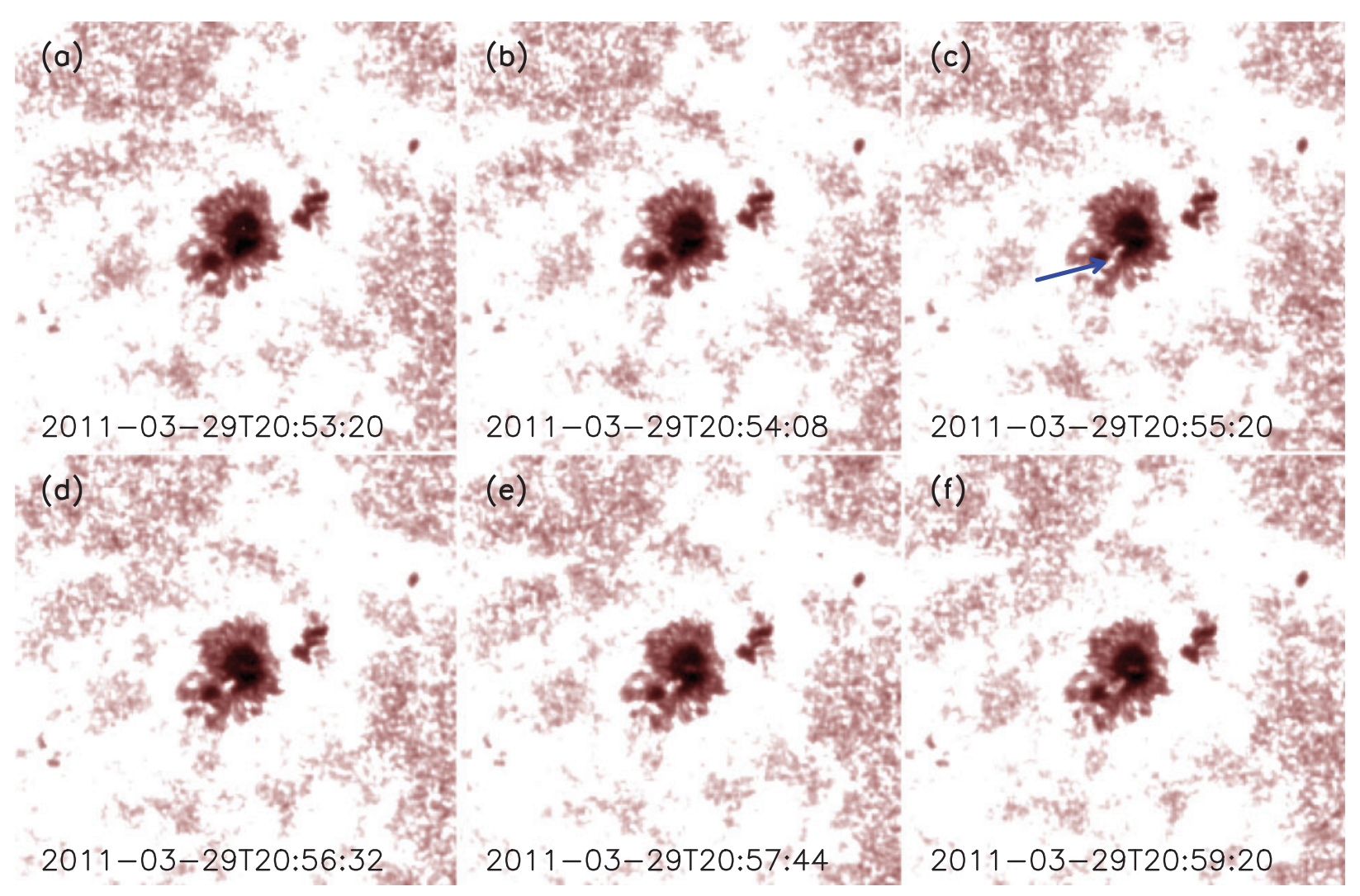

Figure 3 The evolution of 1700- $\AA$ images from 20:53-20:59 UT; the blue arrow in panel (c) should be caused by the back-flow of the jet. 


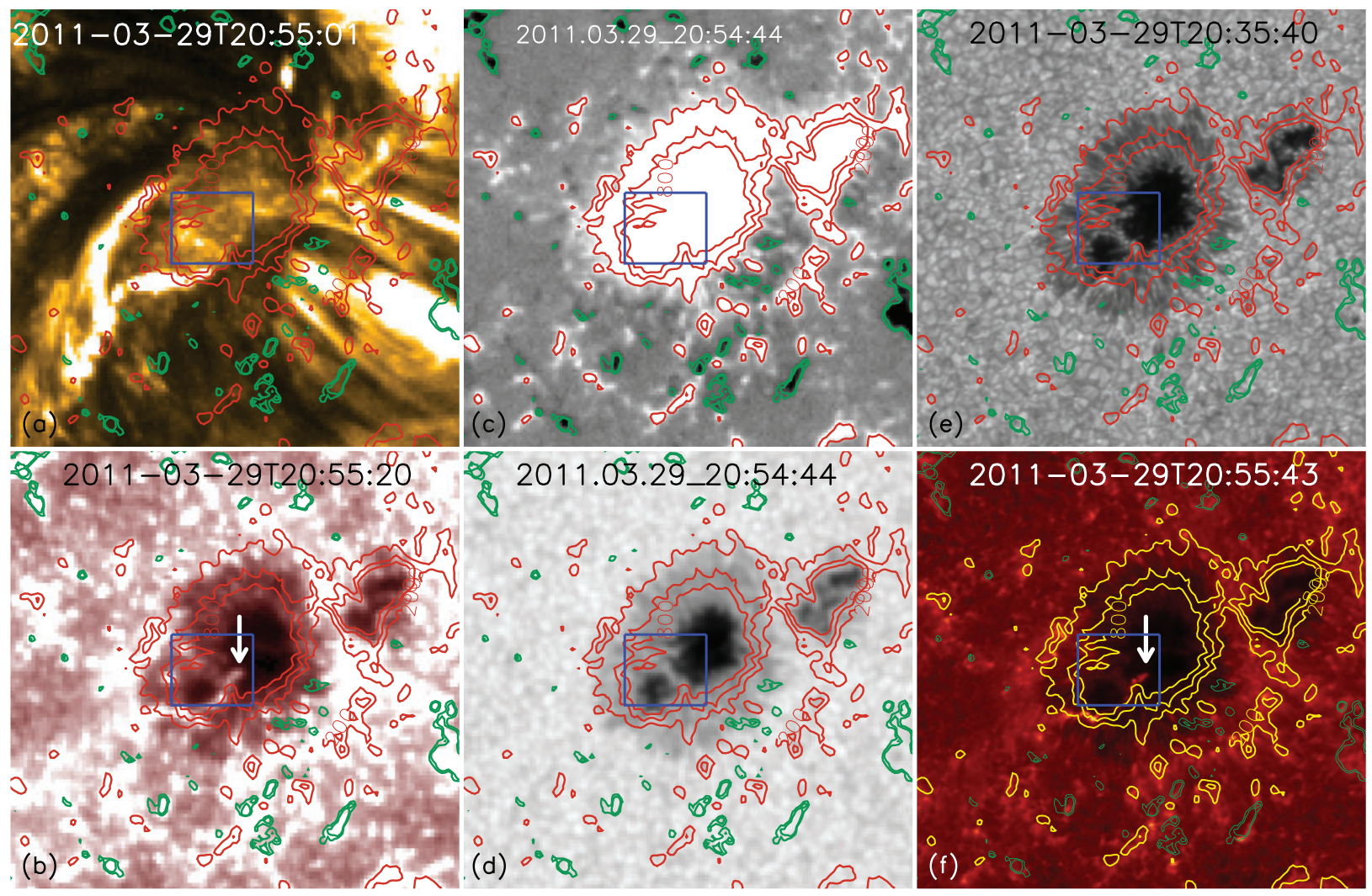

Figure 4 The (a) 171- $\AA$ and (b) 1700- $\AA$ AIA images, (c) LOS magnetogram and (d) continuum intensity HMI images, and (e) G-band and (f) Ca II Hinode images. The contours drawn on each image are line-of-sight magnetic field, where the levels of magnetic field contours are -80 to -50 and $200-800 \mathrm{G}$.
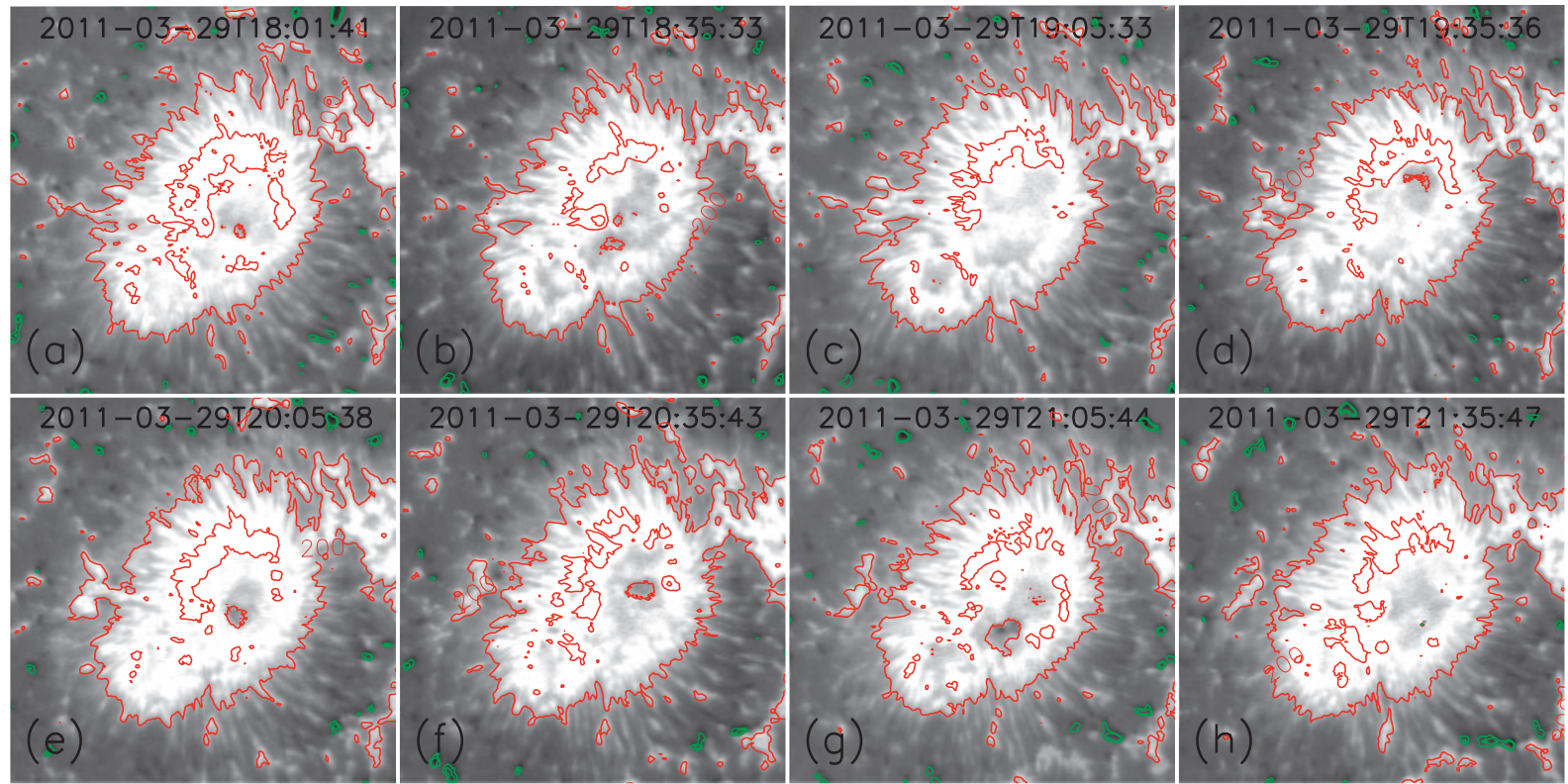

Figure 5 Evolution of the LOS magnetic field observed by SOT/Hinode during 18:01-21:35 UT. The contour levels are from -80 to -50 (green) and 200-800 G (red), and the field of view is $40 \times 40 \mathrm{arcsec}^{2}$. From these images it is found that when comparing the magnetic flux of the main sunspot there is no opposite magnetic flux near the LB.

-80 to -50 and $200-800 \mathrm{G}$, and red/green contours are positive/negative magnetic flux, which is consistent with the HMI LOS magnetic field in Figure 5. It is found that there is no evident negative magnetic flux near the site of jet eruption from the high spatial resolution images of LOS magnetic field.

In order to find the change in the LB during jet ejection, the evolution of the maximal intensity and width of the LB 

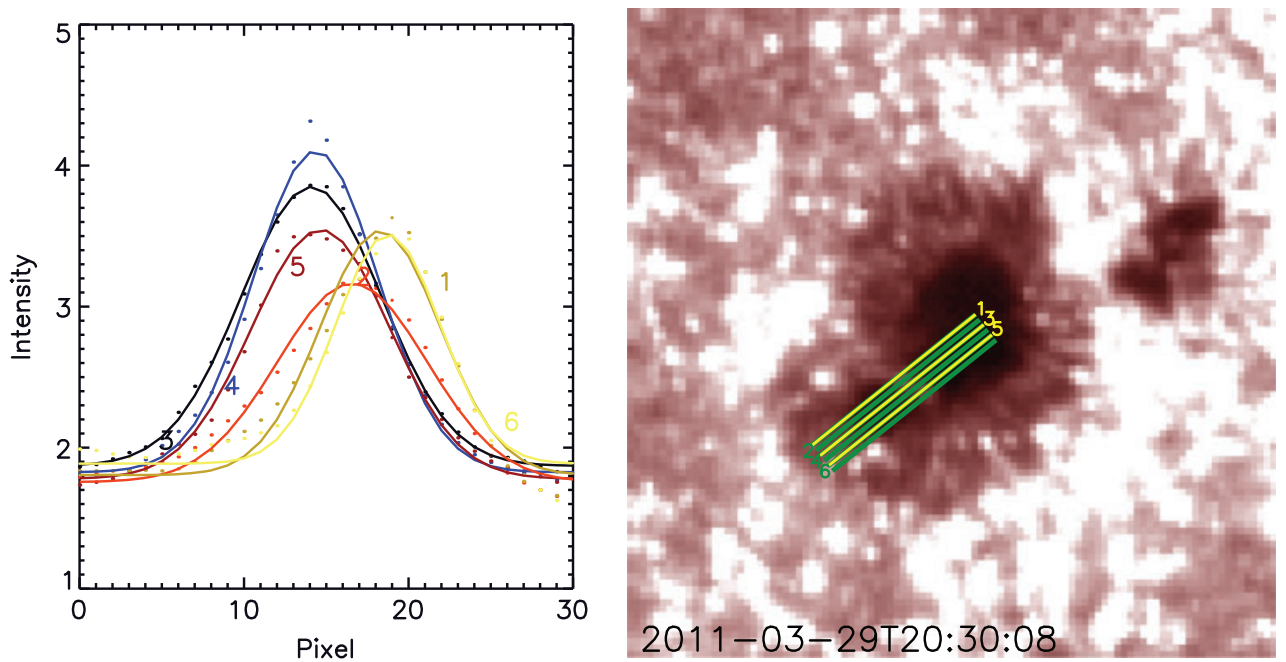

Figure 6 The right frame is an example of a 1700- $\AA$ image and shows six selected slits along the LB. The left frame plots the intensity of the slits and the profile of intensity obtained from a Gaussian fit. The dotted line is the profile of intensity and the solid line is the Gaussian fit. The numbers of slit correspond to those of the labeled profiles of intensity.
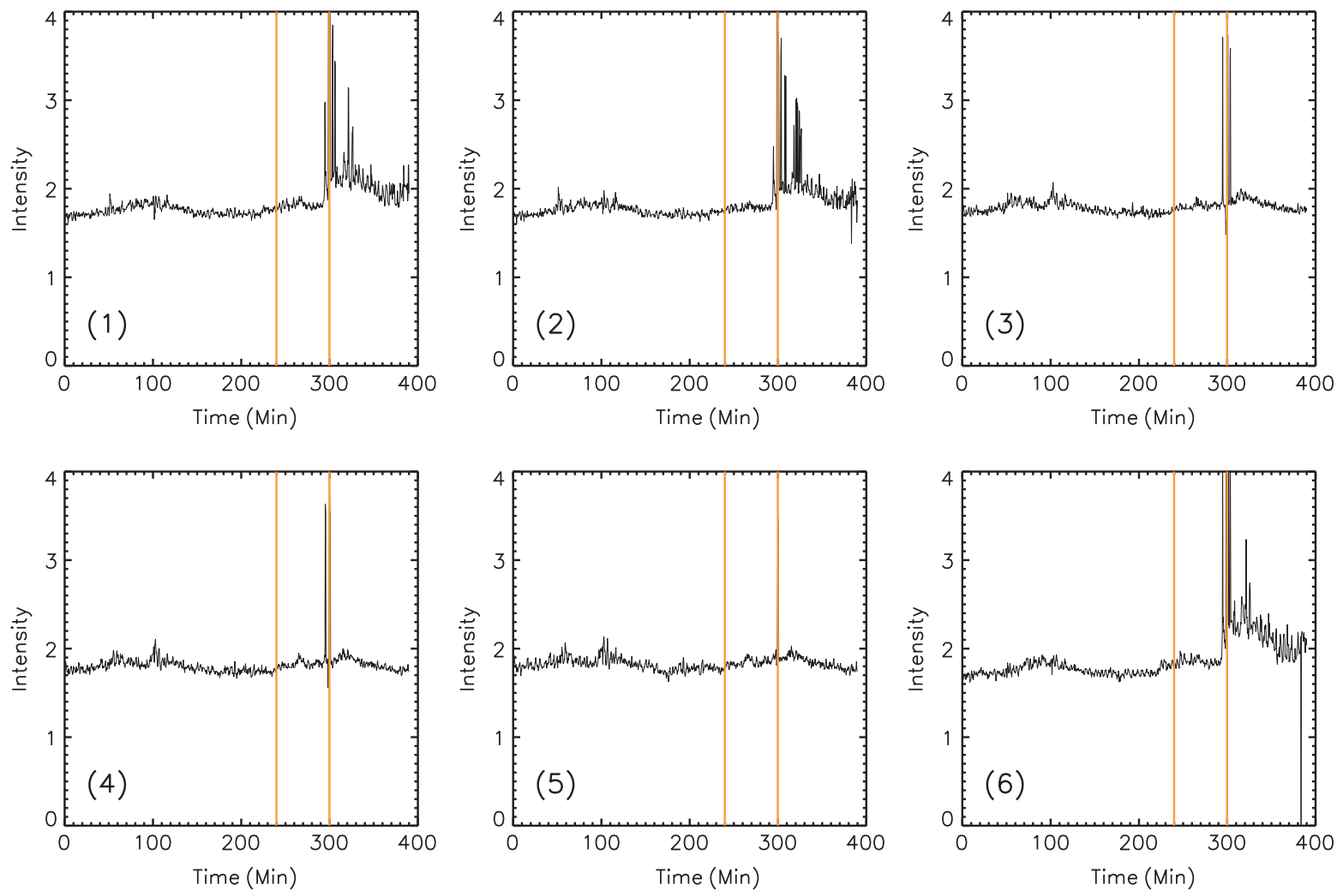

$2011-03-29 \quad 16: 00--22: 30$

Figure 7 The evolution of maximal intensity along the LB obtained from a Gaussian fit of six slits in the AIA 1700- $\AA$ images. The time interval between the two yellow vertical lines is 20:00-21:00 UT.

are studied using $1700-\AA$ images. Six slits are selected along the LB (the right image of Figure 6, which is an example observed at time 20:30:08 UT). The profile of intensity of each slit is fitted using a Gaussian function, then the half-width and height of the Gaussian function are the width and maximal intensity of the LB, respectively (in the left image of Figure 6, the dotted line is the profile of intensity and the solid line is a Gaussian fit to the intensity; the numbers of slits correspond to those of the profile of intensity labeled using different colors). It is found that the results of a Gaussian fit are reasonable and acceptable, because there is no evident deviation between the true profiles and the fitted profile.

Using the above method to calculate a series of $1700-\AA$ images, the evolution of the maximal intensity and width of the LB are plotted in Figures 7 and 8, respectively, 

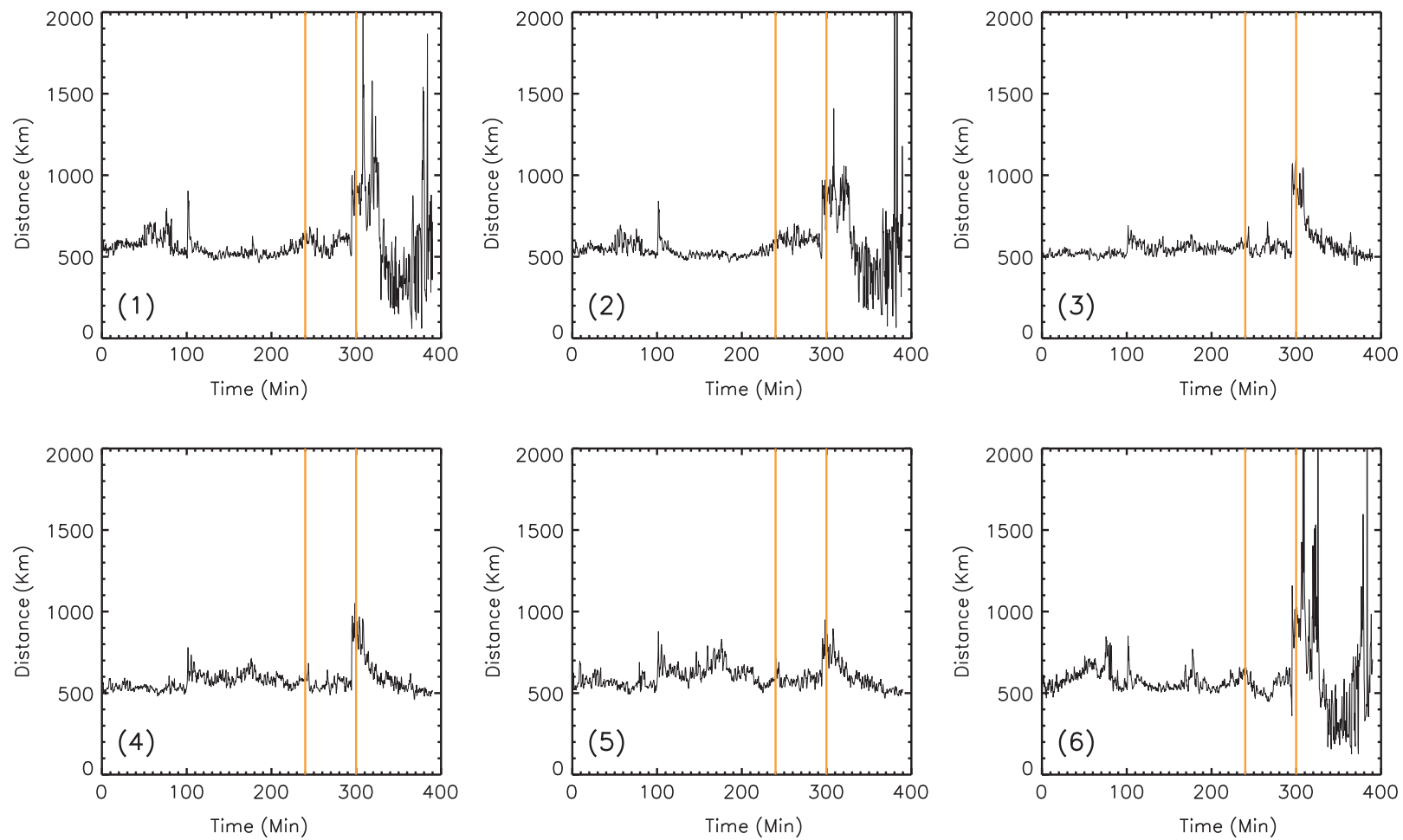

2011-03-29 16:00--22:30

Figure 8 The evolution of width of the LB obtained from a Gaussian fit of six slits in the AIA 1700- $\AA$ images. The time interval between the two yellow vertical lines is 20:00-21:00 UT.

during 16:00-22:30 UT. The time interval between the two yellow vertical lines is 20:00-21:00 UT. From Figure 7, it can be found that before jet creation there is no evident change of maximal intensity; by the time of jet onset the maximal intensity begins to increase slightly, but this increase is not evident; during jet ejection the evolution of maximal intensity is different for the six slits: for slits (1), (2), and (3) it increases slightly but not evidently, for slits (4), (5), and (6) it barely changes, however on the whole there is a trend of increase for the maximal intensity during jet ejections; when the jet finishes the maximal intensity reaches a maximum, caused by the back-flow of the jet, after which time it begins to decrease. From Figure 8 , it can be found that before and during jet ejections no evident rule can be obtained, however after the jet finishes there is a jump of width of the LB, which is caused by the back-flow of the jet; it then narrows down quickly to normal width as before. The above results confirm that this jet has its root near the LB and thus interaction between the LB and the main sunspot may be a direct reason for the creation of this jet. The width of the LB $(\approx 500 \mathrm{~km})$ plotted in Figure 8 is consistent with previous results (Louis et al. 2008).

The HMI continuum intensity images are also studied by the same method as above to investigate the effects of the LB on the photosphere during jet eruptions. Similarly to Figure 6, six slits in the continuum intensity image are selected and shown in the right frame of Figure 9, where the observation obtained at time 20:29:59 UT is used as an example. After a Gaussian fit to a series of continuum intensity images, the evolution of maximal intensity and width of the LB on the photosphere are plotted in Figures 10 and 11, respectively. On the whole, the maximal intensities increase slightly before and during jet ejection, then display a decrease after the jet finishes. On the photosphere, a changing trend in the width of the LB cannot be ruled out before and during jet ejection, and different slits show different changes. However, there is a common feature that the width of the LB broadens to some extent after the jet finishes. It is also found that the width of the LB on the photosphere $(\approx 800 \mathrm{~km})$ is broader than that of the $1700-\AA$ images $(\approx 500 \mathrm{~km})$.

\section{Discussion and Conclusions}

Using multi-spectral images observed by the newly launched satellites Hinode and SDO, the evolution of a LB accompanying a coronal jet eruption is studied. It is revealed that this coronal jet (ejected during 20:0021:07 UT on 2011 March 29 with a peak at 20:45 UT) is related to the LB. This suggests that interaction between LB and main umbra not only has a low-atmosphere response (previous studies include $\mathrm{H} \alpha$ surges and coronal loop enhancement) but also includes more dynamic highatmosphere or coronal activity.

The evolution of the LB during jet eruption is studied based on $1700-\AA$ images and photospheric continuum intensity images. On the whole, the intensity and width of the LB show no evident change before and during jet ejection; however there are evident changes after the jet finishes, which means that the LB also has a dynamic 

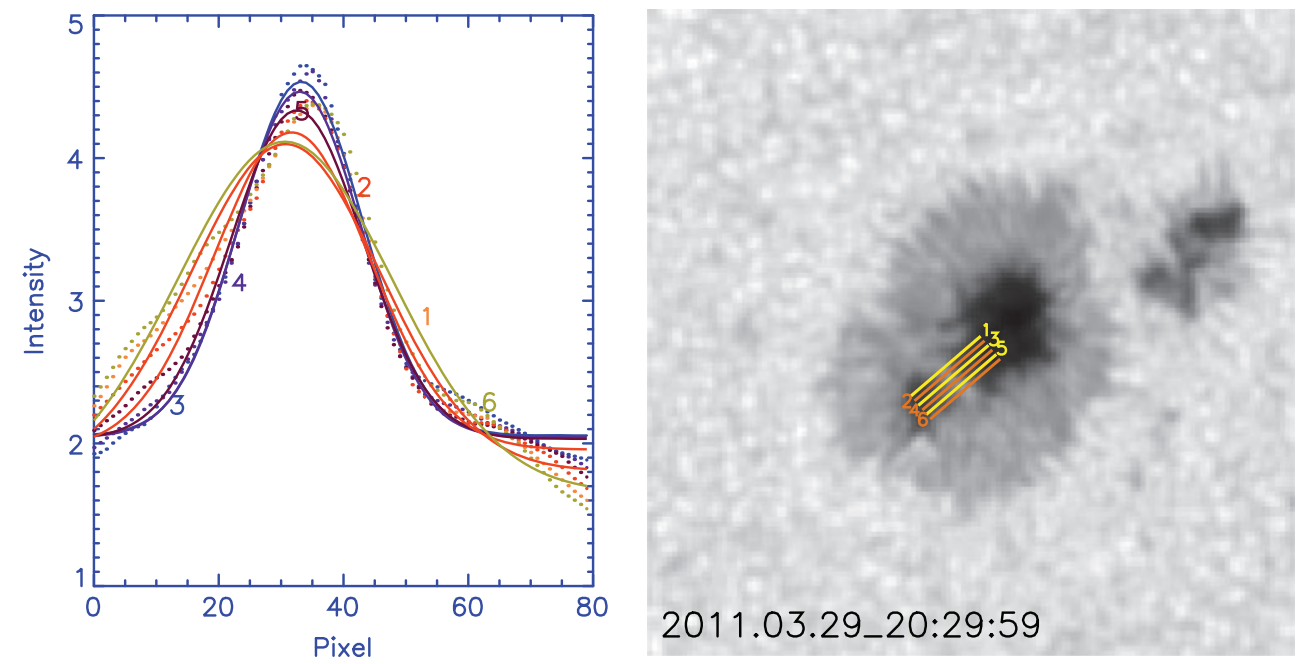

Figure 9 Same as Figure 5, but for HMI continuum intensity.
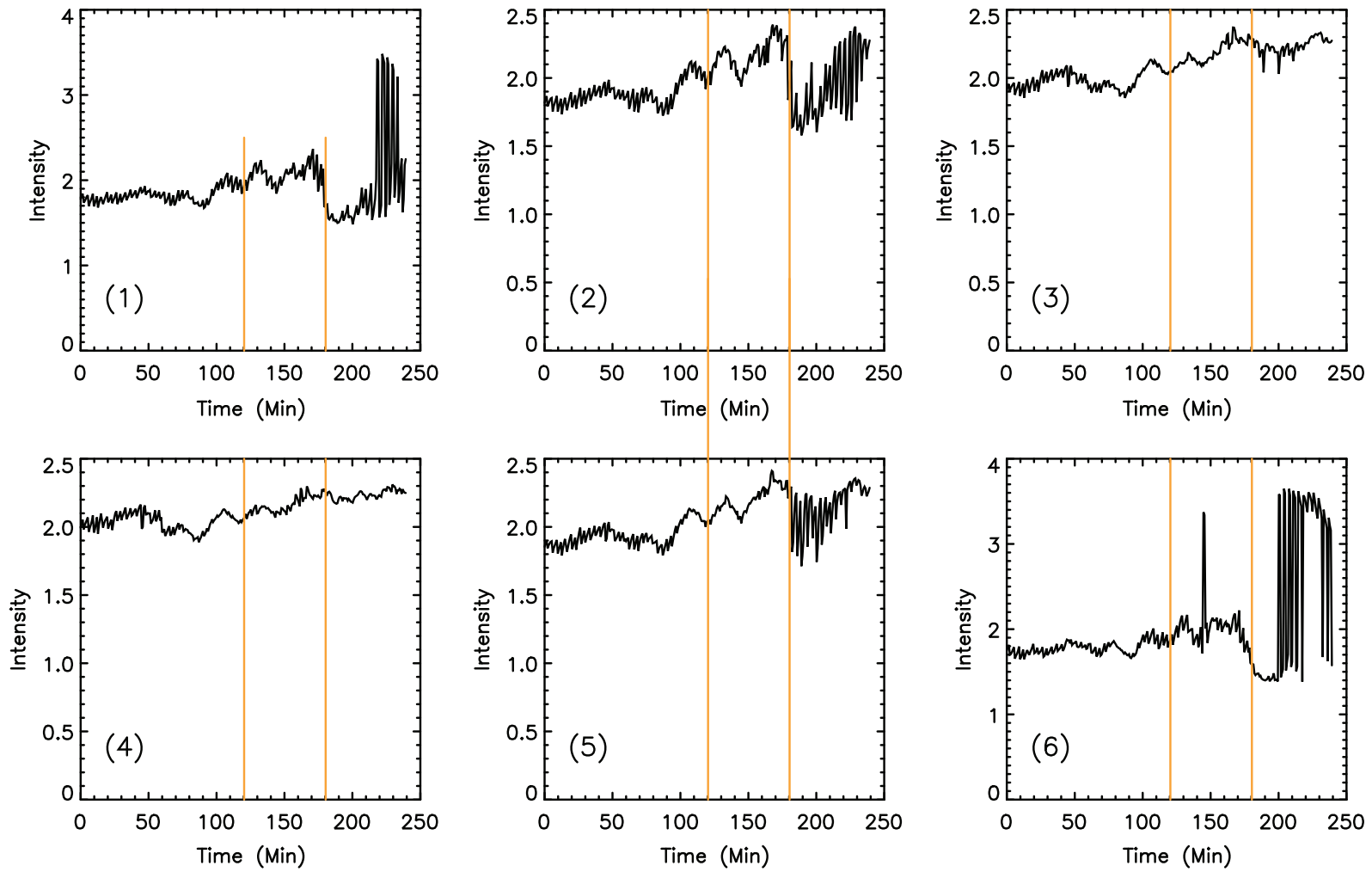

2011-03-29 18:00--21:59

Figure 10 Same as Figure 6, but for HMI continuum intensity.

coronal response and not only a chromospheric response (chromospheric brightening and $\mathrm{H} \alpha$ surges). It is also found that the width of the LB displayed on the photosphere is broader than that displayed on a $1700-\AA$ image. The evolution of intensity and width of the LB at different atmospheric levels must surely give us a changing trend during jet ejection. It is noted that LBs accompanying coronal jets have seldom been studied so far. Thus, it is expected that more scientific data will be made available for similar analysis in the near future.
Normally a jet is regarded as a phenomenon of magnetic reconnection. The existence of opposing magnetic flux is required for reconnection models, but from the line-of-sight magnetic field it is found that there is no evident opposite magnetic flux existing in the region where the jet originated for this event. Thus, there should be magnetic components in the LB that can provide the essential condition for magnetic reconnection. More accurately, the basic condition for magnetic reconnection is an opposite magnetic flux and anomalous resistance. 

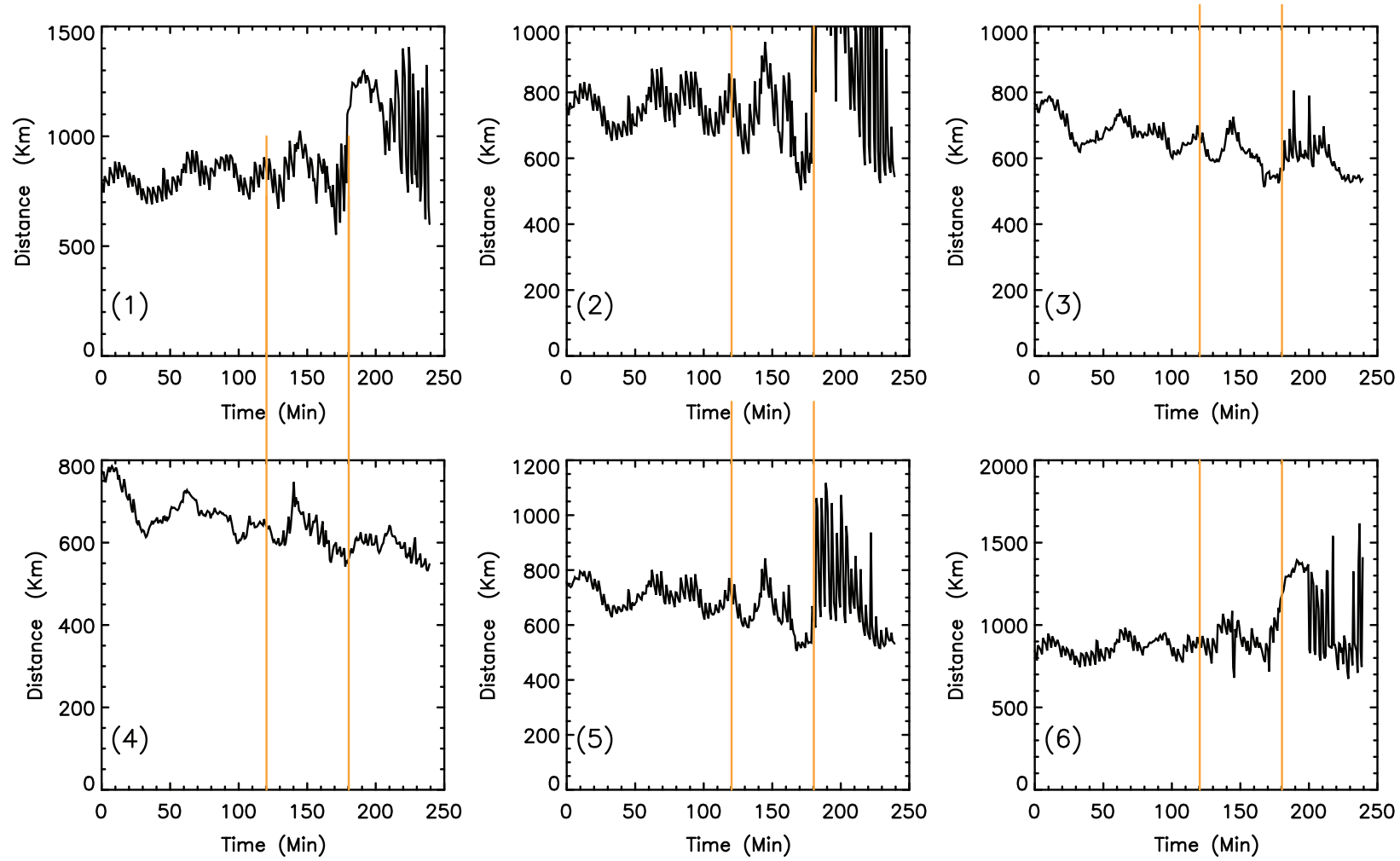

2011-03-29 18:00--21:59

Figure 11 Same as Figure 7, but for HMI continuum intensity.

Generally, the opposite magnetic flux in fact consists of two magnetic topologies and the anomalous resistance is usually caused by some instabilities. Here, the main sunspot and the LB can be considered as two magnetic systems and instability may more easily exist at the boundary between the main sunspot and the LB. For this jet event, the opposite magnetic flux may be below the resolution of the magnetic field observed or, as in Shimizu et al. (2009), a highly twisted magnetic field may be trapped in the LB that can provide the condition for magnetic reconnection. Also, it should be noted that magnetic reconnection does not always require an opposite magnetic flux, for example in component reconnection (there are some angle differences between the two magnetic components). However there may be a deeper physics mechanism for magnetic reconnection that can be studied in future, when we obtain high spatial and temporal resolution vector magnetic fields that can show more of the fine magnetic structure of the LB.

Plasma and magnetic fields fill the entire Sun, and there is a phenomenon of magnetic freezing in regions of strong magnetic field (namely, sunspot regions). Hence, the plasma should flow along magnetic field lines. Sunspot and LB should be considered as two magnetic systems. Previous studies (Ruedi et al. 1979; Leka 1997; Jurcak et al. 2006) show that the magnetic field in a LB is weaker and more inclined than that of the surrounding umbra. For the unipolar sunspot studied here, the magnetic field lines should have a radial shape, and the stronger the magnetic field the more vertical the lines. The LB is another magnetic system, in which the distribution of magnetic field lines should be more inclined. Thus, plasma flows along the field lines of the individual magnetic systems of sunspot and LB. The fine topology of the magnetic field of the LB is unknown to us, due to observing restrictions. Perhaps the field lines lie along the axis of the LB or field lines surround the axis of the LB, hence plasma may flow along the axis of the LB or surround the axis of the LB. Field lines either along the LB or surrounding the LB should be considered as another magnetic system compared with that of the main sunspot, one that is different from the main sunspot. Thus, the basic condition for magnetic reconnection at the boundary between sunspot and LB can exist, and the jet unavoidably becomes the result of magnetic reconnection.

\section{Acknowledgments}

Hinode is a Japanese mission developed and launched by ISAS/JAXA, collaborating with NAOJ as a domestic partner, NASA and STFC (UK) as international partners. Scientific operation of the Hinode mission is conducted by the Hinode science team organized at ISAS/JAXA. This team mainly consists of scientists from institutes in the partner countries. Support for the post-launch operation is provided by JAXA and NAOJ (Japan), STFC (UK), NASA, ESA, and NSC (Norway). This work was partly supported by the National Natural Science Foundation of 
China (Grant Nos. 10611120338, 10673016, 10733020 , 10778723, 11003025, 11103037, 10878016 and 11178016), National Basic Research Program of China (Grant No. 2011CB8114001), Important Directional Projects of Chinese Academy of Sciences (Grant No. KLCX2-YW-T04) and the Young Researcher Grant of National Astronomical Observatories, Chinese Academy of Sciences.

\section{References}

Asai, A., Ishii, T. T. \& Kurokawa, H., 2001, ApJ, 555, L65

Berger, T. E. \& Berdyugina, S. V., 2003, ApJ, 589, L117

Bharti, T., Rimmele, T., Jain, R., Jaaffrey, S. N. A. \& Smart, R. N., 2007, MNRAS, 376, 1291

Boerner, P., Soufli, R., Podgorski, W. \& Wolfson, C. J., 2010, AGUFM, 1871

Bray, R. J. \& Loughhead, R. E., 1964, Sunspots, The International Astrophysics Series (London: Chapman \& Hall)

Garcia de La Rosa, J. I., 1987, SoPh, 112, 49
Jurcak, J., Pillet, V. M. \& Sobotka, M., 2006, A\&A, 112, 49 Katsukawa, Y., 2007, in ASP Conf. Ser. 369, New Solar Physics with Solar-B Mission, ed. K. Shibata, S. Nagata \& T. Sakurai (San Francisco: ASP), 287

Katsukawa, Y., et al., 2007, PASJ, 59, 577

Kosugi, T., et al., 2007, SoPh, 243, 3

Leka, K. D., 1997, ApJ, 484, 900

Louis, P. E., Bayanna, A. R. \& Mathew, S. K., 2008, SoPh, 252, 43 Muller, R., 1979, SoPh, 61, 297

Roy, J.-R., 1973, SoPh, 28, 95

Ruedi, I., Solanki, S. K. \& Livingston, W., 1979, SoPh, 61, 297

Schou, J., Borrero, J. M., Norton, A. A., Tomczyk, S., Elmore, D. \& Card, G. L., 2010, SoPh, 275, 327

Shimizu, T., et al., 2009, ApJ, 696, L66

Sobotka, M., Bonet, J. A. \& Vazquez, M., 1993, ApJ, 415, 832

Sobotka, M., Bonet, J. A. \& Vazquez, M., 1994, ApJ, 426, 404

Spruit, H. C. \& Scharmer, G. B., 2006, ApJ, 415, 832

Title, A., 2010, in 38th COSPAR Scientific Assembly, July 2010 (Bremen), 2

Tsuneta, S., et al., 2008, SoPh, 249, 167

Vasquez, M., 1973, SoPh, 31, 377 